\title{
3-MANIFOLDS WITH NILPOTENT EMBEDDINGS IN $S^{4}$
}

\author{
J.A.HILLMAN
}

\begin{abstract}
We consider embeddings of 3-manifolds $M$ in $S^{4}$ such that the two complementary regions $X$ and $Y$ each have nilpotent fundamental group. If $\beta=\beta_{1}(M)$ is odd then these groups are abelian and $\beta \leq 3$. In general $\pi_{1}(X)$ and $\pi_{1}(Y)$ have 3 -generator presentations, and $\beta \leq 6$. We give two examples illustrating our results.
\end{abstract}

This is a continuation of the papers [4, 5], in which we considered the complementary regions of a closed hypersurface in $S^{4}$. Let $M$ be a closed orientable 3-manifold and $j: M \rightarrow S^{4}=X \cup_{M} Y$ be a locally flat embedding. Then $\chi(X)+\chi(Y)=2$ and we may assume that $\chi(X) \leq$ $\chi(Y)$. In [4, $\S 7]$ we considered the possibilities for $\chi(X), \pi_{X}=\pi_{1}(X)$ and $\pi_{Y}=\pi_{1}(Y)$, and showed that if $\pi_{X}$ is abelian then $\beta=\beta_{1}(M ; \mathbb{Q}) \leq$ 4 or $\beta=6$, while in [5] we attempted to apply 4-dimensional surgery to classify embeddings such that both $\pi_{X}$ and $\pi_{Y}$ are abelian. Here we shall cast our net a little wider. In order to use 4-dimensional surgery arguments we must restrict the possible groups $\pi_{X}$ and $\pi_{Y}$. Under our present understanding of the Disc Embedding Theorem, these groups should be in the class $G$ of groups generated from groups with subexponential growth by increasing unions and extensions [2]. This class includes all elementary amenable groups and is included in the class of restrained groups, those which have no non-cyclic free subgroups. We shall also assume that the embedding $j$ is bi-epic, i.e., that each of the homomorphisms $j_{X *}: \pi=\pi_{1}(M) \rightarrow \pi_{X}$ and $j_{Y *}$ : $\pi \rightarrow \pi_{Y}$ is an epimorphism. This is so if $\pi_{X}$ and $\pi_{Y}$ are nilpotent, since $H_{1}\left(j_{X}\right)$ and $H_{1}\left(j_{Y}\right)$ are always epimorphisms.

We show firstly that if $j$ is bi-epic and $\pi_{X}$ and $\pi_{Y}$ are restrained then $0 \leq \chi(X) \leq \chi(Y)$, so $\chi(X)$ and $\chi(Y)$ are determined by $\beta$, and if $\beta$ is even then $\chi(X)=\chi(Y)=1$ and so $\beta_{2}\left(\pi_{X} ; R\right) \leq \beta_{1}\left(\pi_{X} ; R\right)$ and $\beta_{2}\left(\pi_{Y} ; R\right) \leq \beta_{1}\left(\pi_{Y} ; R\right)$, for any coefficient ring $R$. Secondly, if $\pi_{X}$ and $\pi_{Y}$ are nilpotent then either $\beta=1$ or 3 and $\pi_{X}$ and $\pi_{Y}$ are free abelian

1991 Mathematics Subject Classification. 57N13.

Key words and phrases. embedding, homologically balanced, nilpotent, 3manifold, restrained, surgery. 
groups, or $\beta=0,2,4$ or 6 . If we assume further that $\pi_{X}$ and $\pi_{Y}$ are torsion-free nilpotent groups then there are very few known examples of such groups with Hirsch length $>3$ and balanced presentations. We give two examples illustrating the possibilities allowed by Theorem 2 .

We shall say that an embedding has a group-theoretic property (e.g., abelian, nilpotent, ...) if the groups $\pi_{X}$ and $\pi_{Y}$ have this property.

\section{RESTRAINED EMBEDDINGS}

We begin with an observation that can be construed as a minimality condition.

Lemma 1. Let $J=j_{K, \gamma}$ be an embedding obtained from $j: M \rightarrow S^{4}$ by a proper 2-knot surgery using the 2-knot $K$ and the loop $\gamma \in \pi_{X(j)}$. Then $J$ is not bi-epic, and $\pi_{X(J)}$ is not restrained, unless $\pi_{X(J)}$ is itself a restrained 2 -knot group, in which case $\beta=\beta_{1}(M ; \mathbb{Q})=1$ or 2 .

Proof. Let $C \cong \mathbb{Z} / q \mathbb{Z}$ be the subgroup of $\pi_{X(j)}$ generated by $\gamma$, and let $t$ be a meridian for the knot group $\pi K$. Then

$$
\pi_{X(J)} \cong \pi_{X(j)} *_{C} \pi K /\left\langle\left\langle t^{q}\right\rangle\right\rangle .
$$

Since the 2-knot surgery is proper, $\left\langle\left\langle t^{q}\right\rangle\right\rangle$ is a proper normal subgroup of $\pi K$. Since the image of $\pi_{1}(M)$ lies in $\pi_{X(j)}$, the embedding $J$ cannot be bi-epic. Moreover $\pi_{X(J)}$ can only be restrained if $\pi_{X(j)} \cong \mathbb{Z}$, in which case $\pi_{X(J)} \cong \pi K$ and $\chi(X(j))=0$ or 1 , and so $\beta=1$ or 2 .

Let $G$ be a group. Then $G^{\prime}$ and $\zeta G$ shall denote the commutator subgroup and centre of $G$, respectively.

If $G$ is finitely generated and restrained then $\operatorname{def}(G) \leq 1$. If $\operatorname{def}(G)=$ 1 then $G$ is an ascending HNN extension, and so the first $L^{2}$-Betti number $\beta_{1}^{(2)}(G)=0$. Hence g.d.G $\leq 2$, by [3, Theorem 2.5]. The argument is homological, and so it suffices that the augmentation ideal in $\mathbb{Z}[G]$ have a presentation of deficiency 1 as a $\mathbb{Z}[G]$-module. A finitely generated group $G$ is balanced if it has deficiency $\geq 0$, and is homologically balanced if $\beta_{2}(G ; R) \leq \beta_{1}(G ; R)$, for any coefficient ring $R$.

Let $B S(1, m)$ be the Baumslag-Solitar group with presentation $\langle t, a|$ tat $\left.^{-1}=a^{m}\right\rangle$, for $m \in \mathbb{Z} \backslash\{0\}$. Then $B S(1,1) \cong \mathbb{Z}^{2}$, while $B S(1,-1) \cong$ $\pi_{1}(K b)$ is the Klein bottle group.

Theorem 2. Let $j: M \rightarrow S^{4}$ be a bi-epic embedding such that $\pi_{X}$ and $\pi_{Y}$ are restrained.

If $\beta=\beta_{1}(M ; \mathbb{Q})$ is odd then $\chi(X)=0$ and $\chi(Y)=2$, and $X$ is aspherical. If, moreover, $\pi_{X}$ is almost coherent or elementary amenable then $\pi_{X} \cong \mathbb{Z}$ or $B S(1, m)$, for some $m \neq 0$, and $\beta=1$ or 3 . 
If $\beta$ is even then $\chi(X)=\chi(Y)=1$, and $\pi_{X}$ and $\pi_{Y}$ are homologically balanced.

Proof. If $\pi_{X}$ and $\pi_{Y}$ are each restrained then $\chi(X), \chi(Y) \geq 0$. Hence $(\chi(X), \chi(Y))$ is determined by the parity of $\beta$, since $0 \leq \chi(X) \leq$ $\chi(Y) \leq 2$ and $\chi(X) \equiv \chi(Y) \equiv 1+\beta \bmod (2)$.

Since $j$ is bi-epic, c.d. $X \leq 2$ and c.d.Y $\leq 2$, by [4, Theorem 5.1]. Hence if $\chi(X)=0$ and $\pi_{X}$ is restrained then $X$ is aspherical, by [3, Theorem 2.5]. If, moreover, $\pi_{X}$ is elementary amenable or almost coherent then $\pi_{X} \cong \mathbb{Z}$ or $B S(1, m)$ for some $m \neq 0$, by [3, Corollary 2.6.1]. Hence $\beta=\beta_{1}(X ; \mathbb{Q})+\beta_{2}(X ; \mathbb{Q})=1$, if $\pi_{X} \neq B S(1,1)=\mathbb{Z}^{2}$, and $\beta=3$ if $\pi_{X} \cong \mathbb{Z}^{2}$.

Since $c . d . X \leq 2$ and $X$ is homotopy equivalent to a finite complex the cellular chain complex $C_{*}\left(X ; \mathbb{Z}\left[\pi_{X}\right]\right)$ is chain homotopy equivalent to a finite free complex $C_{*}$ of length 2 , with $C_{0}$ of rank 1 . If $\beta$ is even then $\chi(X)=1$, and so $C_{1}$ and $C_{2}$ have the same rank. Hence $\pi_{X}$ is homologically balanced. Similarly for $\pi_{Y}$.

The latter part of the argument shows that the augmentation ideal in $\mathbb{Z}\left[\pi_{X}\right]$ has a square presentation matrix (i.e., has a presentation of deficiency 0 as a $\mathbb{Z}[G]$-module). We do not know whether the group $\pi_{X}$ must have deficiency $\geq 0$.

There are examples of each type. (See below). There is also a partial converse. If $\chi(X)=0$ and $\pi_{X} \cong B S(1, m)$ for some $m \neq 0$ then $X$ is aspherical and $j_{X *}$ is an epimorphism, by [4, Theorem 5.1].

\section{NILPOTENT EMBEDDINGS}

Nilpotent embeddings are always bi-epic, since homomorphisms to a nilpotent group which induce epimorphisms on abelianization are epimorphisms.

Theorem 3. Let $j: M \rightarrow S^{4}$ be an embedding such that $\pi_{X}$ and $\pi_{Y}$ are nilpotent.

If $\beta=\beta_{1}(M ; \mathbb{Q})$ is odd and $\pi_{X}$ and $\pi_{Y}$ are nilpotent then either $X \simeq S^{1}$ and $Y \simeq S^{2}$ or $X \simeq T$ and $Y \simeq S^{1} \vee S^{2} \vee S^{2}$.

If $\beta$ is even then $\beta=0,2,4$ or 6 , and $\pi_{X}$ and $\pi_{Y}$ are each 3 -generated.

Proof. Suppose first that $\beta$ is odd. Then $X$ is aspherical, since $\chi(X)=$ 0 and $\pi_{X}$ is nilpotent. Hence $\pi_{X} \cong \mathbb{Z}$ or $\mathbb{Z}^{2}$, since c.d. $X \leq 2$. Since $\pi_{Y}$ is nilpotent and $H_{1}(Y ; \mathbb{Z}) \cong H^{2}(X ; \mathbb{Z})=0$ or $\mathbb{Z}, \pi_{Y}=1$ or $\mathbb{Z}$. The further details in this case are given in [5, Theorem 14].

We may assume that $\beta$ is even and $\beta>4$. Since $\chi(X)=1$ and $H_{i}(X ; R)=0$ for $i>2$, we have $\beta_{2}(X ; R)=\beta_{1}(X ; R)$, and so $\beta_{2}\left(\pi_{X} ; R\right) \leq$ $\beta_{1}\left(\pi_{X} ; R\right)$, for any coefficient ring $R$. Since $\pi_{X}$ is finitely generated 
and nilpotent, there is a prime $p$ such that $\pi_{X}$ can be generated by $d=\beta_{1}\left(\pi_{X} ; \mathbb{F}_{p}\right)$ elements. Let $\widehat{\pi_{X}}$ be the pro- $p$ completion of $\pi_{X}$. Since $\pi_{X}$ is nilpotent, it is $p$-good, and so $\beta_{i}\left(\widehat{\pi_{X}} ; \mathbb{F}_{p}\right)=\beta_{i}\left(\pi_{X} ; \mathbb{F}_{p}\right)$, for all $i$. The group $\widehat{\pi_{X}}$ is a pro- $p$ analytic group, and so has a minimal presentation with $d=\beta_{1}\left(\widehat{\pi_{X}} ; \mathbb{F}_{p}\right)$ generators and $r=\beta_{2}\left(\widehat{\pi_{X}} ; \mathbb{F}_{p}\right)$ relators. Since $\beta>2, \widehat{\pi_{X}} ¥ \widehat{\mathbb{Z}}_{p}$, and so $r>\frac{d^{2}}{4}$, by [8, Theorem 2.7]. (Similarly for $\pi_{Y}$.) Therefore $d \leq 3$ and $\beta \leq 2 d \leq 6$.

If $\pi_{X}$ is nilpotent and $\beta_{1}(X ; \mathbb{Q})=0$ then $\pi_{X}$ is finite, while if $\beta_{1}(X ; \mathbb{Q})=1$ then $\pi_{X} \cong F \rtimes \mathbb{Z}$, where $F$ is finite. Thus if $\pi_{X}$ and $\pi_{Y}$ are torsion-free nilpotent and $\beta \leq 3$ then $\pi_{X}$ and $\pi_{Y}$ are abelian. See [5, Theorems 10, 11 and 16] for more on such embeddings.

It is reasonable to restrict consideration further to torsion-free nilpotent groups, as such groups satisfy the Novikov conjecture, and the surgery obstructions are maniable.

If $G$ is torsion-free nilpotent of Hirsch length $h$ then $c . d . G=h$. The first non-abelian examples are the $\mathbb{N} i l^{3}$-groups $\Gamma_{q}$, with presentations $\left\langle x, y, z \mid[x, y]=z^{q},[x, z]=[y, z]=1\right\rangle$. Some of the argument of $[5$, Theorem 18] for the group $\mathbb{Z}^{3}$ extends to the groups $\Gamma_{q}$. The homology of the pair $(X, M)$ with coefficients $\mathbb{Z}\left[\pi_{X}\right]$ gives an exact sequence

$$
H_{2}\left(X ; \mathbb{Z}\left[\pi_{X}\right]\right) \rightarrow H^{2}\left(X ; \mathbb{Z}\left[\pi_{X}\right]\right) \rightarrow H_{1}\left(M ; \mathbb{Z}\left[\pi_{X}\right]\right) \rightarrow 0 .
$$

Let $K_{X}=\operatorname{Ker}\left(j_{X *}\right)=H_{1}\left(M ; \mathbb{Z}\left[\pi_{X}\right]\right)$ and $P=H_{2}\left(X ; \mathbb{Z}\left[\pi_{X}\right]\right)$. Since c.d. $X \leq 2$ and c.d. $\Gamma_{q}=3$ we see that $P$ is a projective $\mathbb{Z}\left[\Gamma_{q}\right]$-module of rank 1 . It is stably free since $\widetilde{K}_{0}(\mathbb{Z}[G])=0$ for torsion-free poly-Z groups $G$, and $P$ has rank 1 since $\chi(X)=1$. Since $E x t_{\left.\mathbb{Z}\left[\pi_{X}\right]\right)}^{i}\left(\mathbb{Z}, \mathbb{Z}\left[\pi_{X}\right]\right)=$ 0 for $i \leq 2$ we then see that $H^{2}\left(X ; \mathbb{Z}\left[\pi_{X}\right]\right) \cong P^{\dagger}=\overline{H_{o m_{\left.\mathbb{Z}\left[\pi_{X}\right]\right)}}\left(P, \mathbb{Z}_{\left[\pi_{X}\right]}\right]}$, and so is also stably free of rank 1 . We thus have an exact sequence

$$
P \rightarrow P^{\dagger} \rightarrow K_{X} \rightarrow 0
$$

However it is not clear that this is as potentially useful as the analogous conditions on abelian embeddings in Theorems 10, 12, 14 and 18 of [5]. Moreover, if $G$ is a nonabelian poly-Z group then there are infinitely many isomorphism classes of stably free $\mathbb{Z}[G]$-modules $P$ such that $P \oplus \mathbb{Z}[G] \cong \mathbb{Z}[G]^{2}[1$. We do not know which can be realized as $H_{2}\left(X ; \mathbb{Z}\left[\pi_{X}\right]\right)$, for an embedding $j$ with $\pi_{X} \cong \Gamma_{q}$. (This contrasts strongly with the case $\pi_{X} \cong \mathbb{Z}^{3}$, for then $P$ is a free module.)

It can be shown that there is just one homologically balanced torsion free nilpotent group with Hirsch length $h=4$, and none with $h=5$ or with $h=6$ and $\beta=3[6]$. The example with $h=4$ is an extension of $\mathbb{Z}^{2}$ by $\mathbb{Z}^{2}$ with presentation

$$
\langle t, u \mid[t,[t,[t, u]]]=[u,[t, u]]=1\rangle .
$$


If we consider more general solvable groups, we can find many metabelian groups of Hirsch length 5 with balanced presentations. One such group has presentation

$$
\left\langle t, x \mid t^{4} x t^{-4}=t^{2} x^{2} t^{-1} x^{-1} t^{-1} x^{-1}, x t^{2} x t^{-2}=t^{2} x t^{-2} x\right\rangle .
$$

This example is the group of a Cappell-Shaneson 3-knot, with commutator subgroup $\mathbb{Z}^{4}$. If $G$ is torsion-free nilpotent and $h(G)>>5$ is $\operatorname{def}(G)<0$ ? In particular, is this so if $h(G) \geq 6$ and $G / G^{\prime} \cong \mathbb{Z}^{3}$ ?

\section{EXAMPLES}

Pairs of groups with balanced presentations and isomorphic abelianizations can be realized by embeddings of 3-manifolds [7].

Our examples are based on 3- and 4-component links $L=L_{a} \cup L_{u} \cup L_{v}$ or $L_{a} \cup L_{b} \cup L_{u} \cup L_{v}$, where $L_{a} \cup L_{b}$ and $L_{u} \cup L_{v}$ are trivial links. The 3-manifold $M$ obtained by 0 -framed surgery on $L$ embeds in $S^{4}$, and the complementary regions have Kirby-calculus presentations in which one of these sublinks is 0-framed and the other dotted (the roles being exchanged for the two regions). The components $L_{a}$ and $L_{b}$ represent words $A$ and $B$ in $F(u, v)$ and the components $L_{u}$ and $L_{v}$ represent words $U$ and $V$ in $F(a, b)$, and we may arrange that $\pi_{X}$ and $\pi_{Y}$ have presentations $\langle u, v \mid A, B\rangle$ and $\langle a, b \mid U, V\rangle$, respectively. (See the Figure.) The embeddings constructed in this way are always bi-epic, since $\pi_{X}$ and $\pi_{Y}$ are generated by the images of meridians of $L$. We shall use the tabulation of links in [9].

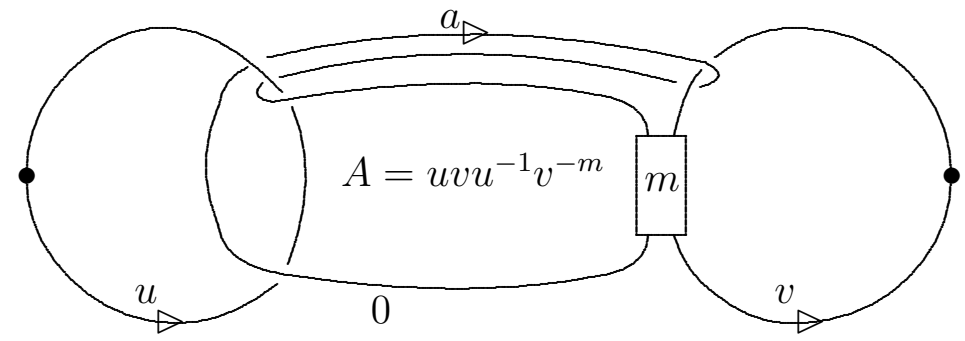

Figure 1

For example, consider the 3-component link in Figure 1, in which the strands in the box have $m$ full twists, $A=u v u^{-1} v^{-m}, B=U=1$ and $V=a^{m-1}$.

When $m=0$ the link is the split union of an unknot and the Hopf link $2_{1}^{2}, M \cong S^{2} \times S^{1}, X \cong D^{3} \times S^{1}$ and $Y \cong S^{2} \times D^{2}$. When $m=1$ the link is the Borromean rings $6_{2}^{3}$, and $X$ is a regular neighbourhood of the unknotted embedding of the torus $T$ in $S^{4}$. When $m=-1$, the link is $8_{9}^{3}$, and $X$ is a regular neighbourhood of the unknotted embeding 
of the Klein bottle $K b$ in $S^{4}$ with normal Euler number 0. In general, $X$ is aspherical, $\pi_{X} \cong B S(1, m)$ and $\pi_{Y} \cong \mathbb{Z} /(m-1) \mathbb{Z}$. (Note however that the boundary of a regular neighbourhood of the Fox 2-knot with group $B S(1,2)$ gives an embedding of $S^{2} \times S^{1}$ with $\pi_{X} \cong B S(1,2)$ and $\chi(X)=0$, but this embedding is not bi-epic and $X$ is not aspherical.)

We may also construct embeddings such that $\pi_{X} \cong B S(1, m)$ and $\chi(X)=1$, while $\pi_{Y} \cong B S(1, m)$ or $\mathbb{Z} \oplus \mathbb{Z} /(m-1) \mathbb{Z}$. These require 4-component links.

This is also the case if $\pi_{X} \cong \Gamma_{q}$, for then $\beta_{2}(X)=\beta_{1}(X)=2$. If $\pi_{Y}$ is abelian then $\pi_{Y} \cong \mathbb{Z}^{2}[4$, Theorem 7.1], and so $q=1$.

It is easy to find a 4-component link $L=L_{a} \cup L_{b} \cup L_{u} \cup L_{v}$ with each 2-component sublink trivial, and such that $L_{a}$ and $L_{b}$ represent (the conjugacy classes of) $A=[u,[u, v]]$ and $B=[v,[u, v]]$ in $F(u, v)$, respectively, while $L_{u}$ and $L_{v}$ have image 1 in $F(a, b)$. Arrange the link diagram so that $L_{u}$ is on the left, $L_{v}$ on the right, $L_{a}$ at the top and $L_{b}$ at the bottom. We may pass one bight of $L_{a}$ which loops around $L_{u}$ under a similar bight of $L_{b}$, so that $U$ now represents $[a, b]$ in $F(a, b)$. Finally we use claspers to modify $L_{u}$ and $L_{v}$ so that they represent $[b, v]$ in $F(b, v)$ and $[a, u]$ in $F(a, u)$. We obtain the link of Figure 2.

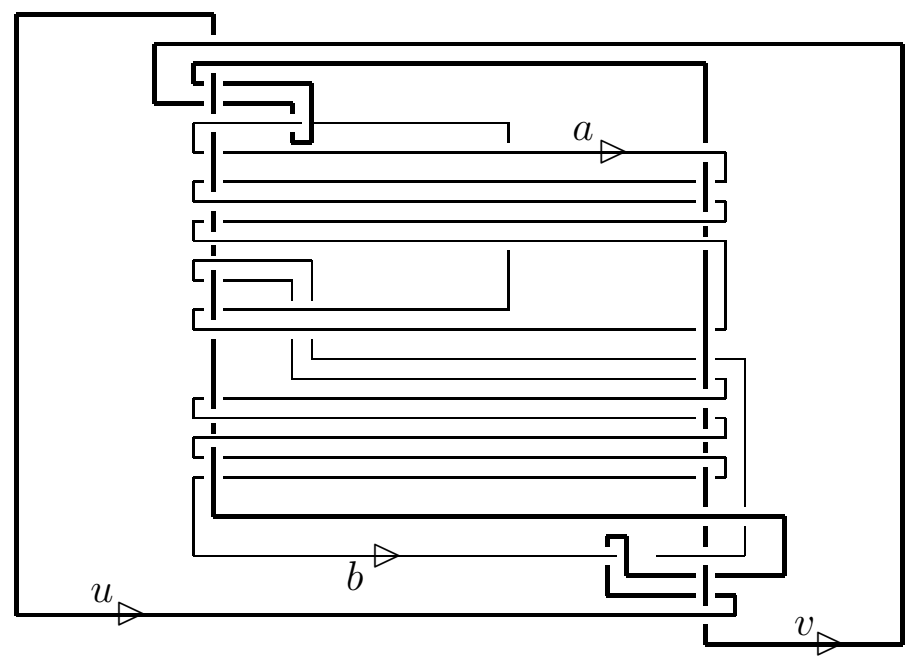

Figure 2

This link may be partitioned into two trivial links in three distinct ways, giving three embeddings of the 3-manifold obtained by 0 -framed surgery on $L$. If the two sublinks are $L_{a} \cup L_{b}$ and $L_{u} \cup L_{v}$ then

$$
A=v u^{-1} v^{-1} u^{-1} v u v^{-1} u, B=v u v^{-1} u^{-1} v^{-1} u v u^{-1}
$$




$$
U=b^{-1} a b a^{-1} \text { and } \quad V=1 .
$$

Hence $\pi_{X} \cong \Gamma_{1}$ and $\pi_{Y} \cong \mathbb{Z}^{2}$.

Each of the other partitions determine abelian embeddings, with $\pi_{X} \cong \pi_{Y} \cong \mathbb{Z}^{2}$ and $\chi(X)=\chi(Y)=1$.

With a little more effort, instead of passing just one bight of $L_{a}$ under $L_{b}$ (as above), we may interlace the loops of $L_{a}$ and $L_{b}$ around each of $L_{u}$ and $L_{v}$ so that $u$ and $V$ represent $[a,[a, b]]$ and $[b,[a, b]]$, respectively, and so that each 2-component sublink of $L$ is still trivial. If we then use claspers again we may arrange that $u$ represents $[a, v]$ and $v$ represents $[b, u]$, so that we obtain a 3 -manifold which has one embedding with $\pi_{X} \cong \pi_{Y} \cong \Gamma_{1}$ and another with $\pi_{X} \cong \pi_{Y} \cong \mathbb{Z}^{2}$. Can we refine this construction so that the third embedding has $\pi_{X} \cong \Gamma_{1}$ and $\pi_{Y} \cong \mathbb{Z}^{2}$ ?

Acknowledgment. This work was begun at the BIRS conference on "Unifying Knot Theory in Dimension 4", 4-8 November 2019.

\section{REFERENCES}

[1] Artamanov, V. A. Projective nonfree modules over group rings of solvable groups, Mat. Sbornik 116 (1981), 232-244.

[2] Freedman, M. H. and Teichner, P. 4-Manifold topology I: subexponential groups, Inventiones Math. 122 (1995), 509-529.

[3] Hillman, J. A. Four-Manifolds, Geometries and Knots, Geometry and Topology Monographs, vol. 5, Geometry and Topology Publications (2002). (Revisions 2007 and 2014).

[4] Hillman, J. A. Complements of connected hypersurfaces in $S^{4}$, Special volume in memory of Tim Cochran, J. Knot Theory Ramif. 2602 (2017), 1740014. (20 pages)

[5] Hillman, J. A. 3-manifolds with abelian embeddings in $S^{4}$, J. Knot Theory Ramif. 2901 (2020), 2050001 (22 pages).

[6] Hillman, J. A. Nilpotent groups with balanced presentations,

[7] Lickorish, W.B.R. Splittings of $S^{4}$, Bol. Soc. Mat. Mexicana 10 (2004), Special Issue, 305-312.

[8] Lubotzky, A. Group presentation, p-adic analytic groups and lattices in $S L_{2}(\mathbb{C})$, Ann. Math. 118 (1983), 115-130.

[9] Rolfsen, D. Knots and Links, Mathematics Lecture Series 7, Publish or Perish, Inc., Berkeley (1976).

School of Mathematics and Statistics, University of Sydney, NSW 2006, Australia

E-mail address: jonathan.hillman@sydney.edu.au 